\title{
The Impact of Macular Pigment Augmentation on Visual Performance Using Different Carotenoid formulations.
}

James Loughman

Technological University Dublin, james.loughman@tudublin.ie

J M. Nolan

Waterford Institute of Technology

Alan Howard

Technological University Dublin

See next page for additional authors

Follow this and additional works at: https://arrow.tudublin.ie/scschphyart

Part of the Optometry Commons

\section{Recommended Citation}

Loughman, J., Nolan JM, Howard A., Connolly, E.,Meagher, K., Beatty, S. (2012) The Impact of Macular Pigment Augmentation on Visual Performance Using Different Carotenoid formulations. Invest Ophthalmal Vis Sci Nov 6. doi:10.1167/iovs.12-10690.

This Article is brought to you for free and open access by the School of Physics \& Clinical \& Optometric Science at ARROW@TU Dublin. It has been accepted for inclusion in Articles by an authorized administrator of ARROW@TU Dublin. For more information, please contact arrow.admin@tudublin.ie, aisling.coyne@tudublin.ie, gerard.connolly@tudublin.ie.

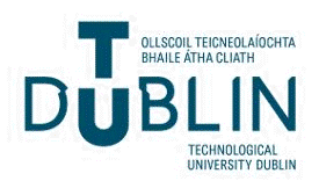




\section{Authors}

James Loughman, J M. Nolan, Alan Howard, E Connolly, K Meagher, and S Beatty

This article is available at ARROW@TU Dublin: https://arrow.tudublin.ie/scschphyart/23 


\title{
The impact of macular pigment augmentation on visual performance in normal subjects: COMPASS
}

\author{
John M. Nolan ${ }^{\mathrm{a}, *, 2}$, James Loughman ${ }^{\mathrm{b}, 2}$, Mukunda C. Akkali ${ }^{\mathrm{a}, 1}$, Jim Stack ${ }^{\mathrm{a}, 2}$, Grainne Scanlon ${ }^{\mathrm{b}, 1}$, \\ Peter Davison ${ }^{\mathrm{b}}$, Stephen Beatty ${ }^{\mathrm{a}, 2}$ \\ a Macular Pigment Research Group, Chemical and Life Sciences, Waterford Institute of Technology, Waterford, Ireland \\ ${ }^{\mathrm{b}}$ Macular Pigment Research Group, Optometry Department, Dublin Institute of Technology, Dublin, Ireland
}

\section{A R T I C L E I N F O}

\section{Article history:}

Received 30 September 2010

Received in revised form 8 December 2010

Available online 13 January 2011

\section{Keywords:}

Macular pigment

Lutein

Zeaxanthin

Vision performance

\begin{abstract}
A B S T R A C T
This study was conducted to investigate whether augmentation of macular pigment (MP) enhances visual performance (VP). 121 normal subjects were recruited. The active (A) group consumed $12 \mathrm{mg}$ of lutein (L) and $1 \mathrm{mg}$ of zeaxanthin (Z) daily. MP optical density (MPOD) was assessed by customized heterochromatic flicker photometry. VP was assessed as best corrected visual acuity (BCVA), mesopic and photopic contrast sensitivity (CS), glare disability, photostress, and subjective visual function. Subjects were assessed at baseline; 3; 6; 12 months (V1, V2, V3 and V4, respectively). Central MPOD increased significantly in the A group $(p<0.05)$ but not in the placebo group $(p>0.05)$. This statistically significant increase in MPOD in the A group was not, in general, associated with a corresponding improvement in VP $(p>0.05$, for all variables), with the exception of a statistically significant time/treatment effect in "daily tasks comparative analysis" $(p=0.03$ ). At V4, we report statistically significant differences in mesopic CS at $20.7 \mathrm{cpd}$, mesopic CS at $1.5 \mathrm{cpd}$ under high glare conditions, and light/dark adaptation comparative analysis between the lower and the upper MP tertile groups $(p<0.05)$ Further study into the relationship between MP and VP is warranted, with particular attention directed towards individuals with low MP and suboptimal VP.
\end{abstract}

(c) 2011 Elsevier Ltd. All rights reserved.

\section{Introduction}

The dietary carotenoids zeaxanthin $(\mathrm{Z})$ and lutein $(\mathrm{L})$ and L's retinal isomer meso-zeaxanthin (meso-Z) are lipid-like molecules that accumulate at the macula, where they are collectively referred to as macular pigment (MP) (Bone, Landrum, Hime, Cains, \& Zamor, 1993). An average western diet contains about $1.3-3 \mathrm{mg} /$ day of $\mathrm{L}$ and Z combined (Nebeling, Forman, Graubard, \& Snyder, 1997a, 1997b), with significantly more $L$ than $Z$ (represented by an estimated ratio of $7: 1$ ). Approximately $78 \%$ of dietary $L$ and $Z$ is sourced from vegetables (Sommerburg, Keunen, Bird, \& van Kuijk, 1998). $\mathrm{L}$ is found in highest concentrations in dark green leafy vegetables, such as spinach, kale, and collard greens (Sommerburg et al., 1998). $Z$ is the major carotenoid found in orange peppers, and oranges, with a high mole percentage of both $\mathrm{L}$ and $\mathrm{Z}$ being found in egg yolk (Sommerburg et al., 1998), with comparable amounts of $\mathrm{L}$ and $\mathrm{Z}$ recently reported in corn and a variety of corn

\footnotetext{
* Corresponding author.

E-mail address: jmnolan@wit.ie (J.M. Nolan).

1 Disclosure: Mukunda C. Akkali, None; Grainne Scanlon, None.

2 James Loughman and Jim Stack do consultancy work for nutraceutical companies in a personal capacity. John M. Nolan and Stephen Beatty do consultancy work for nutraceutical companies, in a personal capacity, and as directors of Nutrasight Consultancy Limited.
}

containing products (e.g. cornmeal and cereal) (Perry, Rasmussen, \& Johnson, 2009). Possible dietary sources of meso-Z include shrimp, certain marine fish, and turtles, none of which are found in a typical western diet (Maoka, Arai, Shimizu, \& Matsuno, 1986), however, it has recently been suggested that MZ may be present in some other, yet to be identified, foods (Connolly et al., 2010).

The macula is a specialized part of the retina, as it mediates central vision, provides sharpest visual acuity, and facilities best color discrimination (Hirsch \& Curcio, 1989). Age-related macular degeneration (AMD) is a disease of the macula and results in the loss of central and color vision. AMD is the most common cause of blindness in the elderly population in the developed world (Congdon et al., 2004). It is now understood that oxidative stress (Beatty, Koh, Henson, \& Boulton, 2000; Winkler, Boulton, Gottsch, \& Sternberg, 1999), exacerbated in part by cumulative shortwavelength visible light exposure (Algvere, Marshall, \& Seregard, 2006; Fletcher et al., 2008), is important in the aetiopathogenesis of AMD. MP is a short-wavelength (blue) light filter (Bone, Landrum, \& Cains, 1992) and a powerful antioxidant (Khachik, Bernstein, \& Garland, 1997), and is therefore believed to protect against AMD (Loane, Kelliher, Beatty, \& Nolan, 2008). This hypothesis, referred to as the "protective" hypothesis of MP, has been studied and reported on extensively (Loane et al., 2008). 
Beyond its "protective" hypothesis, MP's optical and anatomic properties have prompted the "optical" hypotheses of this pigment. The "optical" hypotheses of MP have been previously discussed by Reading and Weale (1974) and later by Nussbaum, Pruett, and Delori (1981), and include MP's putative ability to enhance visual performance and/or comfort by attenuation of the effects of chromatic aberration and light scatter, via its short wave light-filtering properties (Walls \& Judd, 1933). This traditional description of the "optical hypothesis" does not account for additional mechanisms whereby MP may enhance visual performance, that are, perhaps, unrelated to the short wave filtration properties of MP. MP has been shown to exhibit dichroic properties (Hemenger, 1982) which may facilitate the reduction of glare disability through preferential absorption of polarized light. Higher MPOD has also been observed to relate to a trend towards lower root-mean-square wavefront aberrations (in particular, higher order aberrations), thereby enhancing visual performance (Kvansakul et al., 2006).

There is one additional, and important, mechanism, whereby MP may have a beneficial effect on visual performance and experience. The antioxidant properties of the MP carotenoids may attenuate or prevent the deleterious effects of free radical damage on the physiological functions of the photoreceptors and their axons.

Many studies (to date mostly cross-sectional in design) have evaluated, and reported on the role of MP in visual performance, including: visual acuity; contrast sensitivity; glare disability; photostress recovery; critical flicker fusion frequency (CFF); color vision (amongst others) (Bartlett \& Eperjesi, 2008; Engles, Wooten, \& Hammond, 2007; Hammond \& Wooten, 2005; Kvansakul et al., 2006; Loughman et al., 2010; Rodriguez-Carmona et al., 2006; Stringham, Fuld, \& Wenzel, 2004; Stringham \& Hammond, 2007, 2008; Wooten \& Hammond, 2002). However, a placebo-controlled, randomized, L-based supplementation trial was needed to investigate if augmentation of MPOD actually enhances visual performance and/or comfort. The Collaborative Optical Macular Pigment ASsessment Study (COMPASS), presented here, was designed specifically to answer this important research question.

\section{Methods}

\subsection{Subjects and study sites}

COMPASS was conducted at Waterford Institute of Technology (WIT) and Dublin Institute of Technology (DIT), vision science laboratories, located in the southeast and east of the Republic of Ireland, respectively. One hundred and twenty-one healthy subjects volunteered to participate in this two-centered study, which was approved by the research ethics committees at both study sites. Self-selected recruitment of subjects (WIT: $n=61$ and DIT: $n=60$ ) was facilitated by poster and newsletter advertisement, and also by word of mouth, in the respective local communities. Informed consent was obtained from each volunteer, and the experimental procedures adhered to the tenets of the Declaration of Helsinki.

All subjects were aged between 18 to 41 years, in perfect general (self report) and ocular health (see below), and with visual acuity of at least 20/30 in the study eye. A typical study visit lasted approximately $4 \mathrm{~h}$. Subjects were assessed at baseline, three, six, and 12 months (V1, V2, V3 and V4, respectively). All subjects recruited into the study were classed as naïve observers to the tests carried out (with the exception of the visual acuity test, with which all subjects were familiar). However, to optimize performance, and also to minimize any potential learning effects on performance, all subjects underwent a defined period of pre-test training. This training consisted of careful explanation of the nature of each test, pictorial and/or video demonstration of the test requirements and procedure, and was followed by a defined session of pre-test practice.

\subsection{Study design and formulation}

COMPASS is a registered trial on the ISRCTN database (number 35481392), and is a randomized placebo-controlled clinical trial of oral supplementation with a formulation containing the macular carotenoids ( L and Z) and co-antioxidants versus placebo. The tablets used in the current study were hard film coated tablets. The daily dose of two tablets for the active (A) group consisted of $12 \mathrm{mg}$ $\mathrm{L}, 1 \mathrm{mg} \mathrm{Z}$ (provided as ester), $120 \mathrm{mg}$ vitamin C, $17.6 \mathrm{mg}$ vitamin $\mathrm{E}, 10 \mathrm{mg}$ zinc and $40 \mu \mathrm{g}$ selenium. The placebo $(\mathrm{P})$ consisted of cellulose, lactose and magnesium stearate, and was manufactured to be identical to the A preparation in terms of size and color. The study tablets for the $\mathrm{A}$ and $\mathrm{P}$ groups were packaged into identical blister packs which contained the subjects' anonymized unique identification number and COMPASS study label information. Subjects were instructed to consume the daily dose of two tablets with a meal.

Compliance was assessed by tablet counting at each study visit, and encouraged by frequent reminder telephone calls and text messages by the study COMPASS research team. Compliance was also assessed at the end of the study by quantifying $\mathrm{L}$ and $\mathrm{Z}$ concentrations in serum, at each study visit, using high performance liquid chromatography (HPLC).

\subsection{Demographic, medical history, lifestyle and vision case history questionnaires}

The following details were recorded, for each volunteer, on a purpose designed case report form: demographics; general health status; smoking habits (never, current or past); alcohol consumption (average unit weekly intake); exercise (minutes per week); body mass index (BMI, $\mathrm{kg} / \mathrm{m}^{2}$ ); blood pressure; ethnicity; marital status; education; occupation.

A vision case history was also performed, and details reported included: time since last eye examination; spectacles or contact lens use; history of ocular treatment or surgery; history of occlusion therapy or visual training in childhood; family history of eye disease; current problems with vision; asthenopia associated with computer use; history of headaches.

\subsection{Diet and serum concentrations of lutein and zeaxanthin}

Dietary intakes of $\mathrm{L}$ and $\mathrm{Z}$ were quantified using a self-administrated, semiquantitative food frequency questionnaire developed by the Scottish Collaborative Group at the University of Aberdeen (Scotland UK), recently described by O'Connell et al. (O'Connell et al. 2008) Serum concentrations of $L$ and $Z$ were quantified by HPLC using an assay previously reported by Loane et al.(Loane, Nolan, \& Beatty 2010).

\subsection{Spectacle refraction, visual acuity, and ocular dominance}

Each subject underwent precise spectacle refraction by an experienced optometrist to determine refractive error and best corrected visual acuity (BCVA) for each eye. A computer generated LogMAR test chart (Test Chart 2000 Pro; Thomson Software Solutions) was used to determine BCVA at a viewing distance of $4 \mathrm{~m}$, using a Sloan ETDRS letterset. BCVA was determined as the average of three measurements, with letter and line changes facilitated by the software pseudo-randomization feature. Best corrected visual acuity was recorded using a letter-scoring visual acuity rating, with $20 / 20$ visual acuity assigned a value of 100 . Best corrected visual acuity was scored relative to this value, with each letter correctly identified assigned a nominal value of one, so that, for example, a BCVA of $20 / 20^{+1}$ equated to a score of 101 , and $20 / 20^{-1}$ to 99 . The study eye was selected on the basis of ocular dominance, determined using the Miles Test (Roth, Lora, \& Heilman, 2002) with 
the dominant eye chosen as the study eye, except in cases of observed equidominance, in which case the right eye was selected. All subsequent tests were conducted with the subject's optimal subjective refraction in place.

\subsection{Glare disability}

Glare disability is a term used to describe the degradation of visual performance typically caused by loss of retinal image contrast. Glare disability is often caused, for example, by surface light reflections, or bright light sources such as car headlights, and typically is a consequence of increased forward light scatter within the eye. Glare disability was assessed using a Functional Acuity Contrast Test (FACT) (Hitchcock, Dick, \& Krieg, 2004; Terzi, Buhren, Wesemann, \& Kohnen, 2005), displayed using the Functional Vision Analyzer (Hohberger, Laemmer, Adler, Juenemann, \& Horn, 2007) (Stereo Optical Co., Inc., Chicago, IL), which is a desktop device that allows the measurement of contrast sensitivity, and includes a customized internal glare source for assessing the impact of glare on this measure of visual performance. The test comprised linear, vertically oriented, sine wave gratings presented at five different spatial frequencies including 1.5, 3, 6, 12 and 18 cycles per degree (cpd). Nine circular patches were presented at each spatial frequency, the contrast of each patch decreasing by $0.15-\log$ units from the previous. Gratings were tilted $-15^{\circ}, 0^{\circ}$ or $+15^{\circ}$ with respect to the vertical, to keep them within the orientation bandwidth of the visual channel. The background was tapered into a grey field in order to keep retinal illumination constant and avoid ghost imaging. Baseline contrast sensitivity was determined on the basis of the lowest contrast compatible with accurate determination of patch orientation across all five spatial frequencies for mesopic [three candelas per meter squared $\left(\mathrm{cdm}^{-2}\right)$ ] instrument background conditions, initially in the absence of a glare source. Subjects were asked to identify grating orientation, starting with the patch at highest contrast, and continuing until identification was no longer possible due to reducing contrast. Subjects were instructed not to guess, but to respond "don't know" if patch orientation could not be correctly identified. As this procedure represented a non-standard psychophysical method of threshold detection, each subject was required to re-identify the orientation of certain gratings in a pseudo-random fashion in order to confirm the validity of the subject responses at each spatial frequency. Glare disability was assessed using a radial glare source consisting of 12 white LEDs arranged circumferentially in an oval pattern surrounding the grating charts (ranging from $4.5^{\circ}$ to $6^{\circ}$ from central fixation). These LEDs have a color temperature of $6500 \mathrm{~K}$, and the spectral emission profile demonstrated a single large peak at $453 \mathrm{~nm}$ (close to MP peak absorption), where the spectral irradiance was approximately double that of the peak emissions in the flatter emission spectrum across mid to long wavelengths. Two customized intensity settings were used to determine the effect of different levels of glare on contrast sensitivity. Glare source settings were set at a medium intensity of 42 Lux and a higher intensity of 84 Lux. All correct responses were entered into the Eyeview software provided, and contrast sensitivity scores for no glare, medium and high glare conditions were determined for the respective spatial frequencies.

\subsection{Visual Function in Normals questionnaire}

A 30-part, non-validated, Visual Function in Normals questionnaire (VFNq30) was designed specifically for the study (JL). The design was based loosely on a previously validated visual activities questionnaire (Sloane, Ball, Owsley, Bruni, \& Roenker, 1992), but adapted to suit a normal, young and healthy population sample. This questionnaire allowed the subject to quantify their visual performance using three separate metrics: situational analysis (SA) which required the subject to rate their visual performance in specified daily life situations; comparative analysis (CA) which required the subject to compare their perceived visual performance to that of their peers/family/friends; subject satisfaction score (SSS) which required the subject to provide an overall estimate of their perceived quality of vision. Each of the three metrics above was computed to give a performance score for five different functional aspects of their vision: acuity/spatial vision; glare disability; light/dark adaptation; daily visual tasks; color discrimination.

\subsection{Contrast sensitivity function}

A Dell Dimension 9200 computer and a Metropsis Visual Stimulus Generation device (VSG (ViSaGe S/N: 81020197), Cambridge Research Systems Ltd., Cambridge, UK) were used to generate and control the stimuli. The VSG provided 14-bit output resolution per phosphor. The stimuli were displayed on a 19" ViewSonic professional series p227f color CRT flat screen monitor with a frame rate of $119.98 \mathrm{~Hz}$. The resolution of the monitor was set to $1024 \times 769$ pixels. Non-linearities in the screen luminance output were eliminated by gamma correction prior to testing using a photometer system (Opti-Cal; Minolta, Japan). The Metropsis software calculated the inverse curves required to correct for the monitor's non-linearities.

The Metropsis contrast sensitivity system generated luminance modulated sine gratings (Gabor patches). The orientation of the stimuli was vertical. The Gabor patches were presented on the CRT monitor and subtended a visual angle of $4.2^{\circ}$. The mean luminance was used as the background luminance. The Gabor had a two-dimensional spatial Gaussian envelope and was radially symmetrical with equal standard deviations, $\delta x$ and $\delta y$.

Contrast sensitivity functions were determined under both mesopic and photopic conditions. Each subject was seated at a fixed viewing distance of $1.5 \mathrm{~m}$ from the CRT monitor. Natural pupils were used throughout the experiment. The non-dominant eye was occluded. Testing was carried out in a light free (other than CRT background mesopic and photopic light) environment. The subject was dark adapted for $5 \mathrm{~min}$ and a 5 -min training session was given prior to testing under mesopic conditions. Subject responses were recorded using a handheld responder (CR6, Cambridge Research Systems Ltd., Cambridge, UK), which communicated with the VSG device via an infra red link. A four alternate forced choice testing system was used, with four possible target locations. The stimuli were randomly presented at $2^{\circ}$ spatial offset from the central cross target. The subject indicated the location of the target in relation to the fixation cross using the appropriate button on the responder box. The subject's contrast sensitivity was determined for five different spatial frequencies (1.0, 4.1, 7.5, 11.8 and $20.7 \mathrm{cpd})$ under both mesopic and photopic conditions, all at a mean luminance of $3 \mathrm{cdm}^{-2}$ (mesopic) and $100 \mathrm{cdm}^{-2}$ (photopic).

A linear staircase method was used to determine the contrast threshold. The first Gabor at a particular location was presented at an initial contrast level where it was anticipated that the observer would be able to detect the Gabor patch for that particular spatial frequency (initial contrast settings were informed by a brief pilot study involving five young healthy subjects). Subsequently, the contrast of the Gabor patch was varied using an adaptive staircase procedure, which was computer controlled and depended upon the subject's responses. The stimulus contrast was reduced in steps of $0.3 \log$ units until the subject did not detect the Gabor patch (first reversal). The contrast was subsequently increased by 0.15-log unit steps until the subject saw the Gabor patch and responded correctly (second reversal). The Metropsis software calculated the contrast threshold for each location and spatial frequency by taking the mid-point between the mean for peaks and 
troughs for 12 reversal points. The standard deviation was calculated by taking the deviations of the peak reversals from their peak means and using the average square of these deviations to calculate a peak variance. This method was repeated for the troughs. The square root of both variances were then calculated and averaged to provide the threshold standard deviation.

For each subject, the Metropsis software plotted the inverse of the contrast threshold against the range of spatial frequencies tested to provide a contrast sensitivity function under both mesopic and photopic conditions.

\subsection{Photostress recovery}

Photostress recovery time (PRT) was calculated using a macular automated photostress (MAP) test (Dhalla \& Fantin, 2005; Dhalla, Fantin, Blinder, \& Bakal, 2007). MAP is a novel photostress method for the evaluation of macular function using the Humphrey ${ }^{\circledR}$ field analyzer (Model 745i Carl Zeiss Meditec Inc. Dublin, CA, USA). The foveal threshold feature of the field analyzer was used to establish baseline foveal sensitivity as the average of three consecutive foveal sensitivity measurements recorded in decibels $(\mathrm{dB})$, with each $\mathrm{dB}$ representing a $0.1 \mathrm{log}$ unit sensitivity variation.

Following baseline foveal sensitivity calculation, the subject was exposed to a photostress stimulus, which consisted of a 5-s exposure to a 300-W, 230-V tungsten lamp head from a viewing distance of $1 \mathrm{~m}$. The spectral irradiance in the wavelength range, $300-800 \mathrm{~nm}$, was measured using a Bentham DMc 150 double monochromator scanning spectroradiometer. The input optic consisted of a very high precision cosine response diffuser (f2 error $<1 \%$ ) and the measurements were performed in $1 \mathrm{~nm}$ intervals. Calibration was carried out with reference to a quartz-halogen lamp traceable to the UK National Physical Laboratory. The illuminance at $1 \mathrm{~m}$ was obtained by using the photopic weighting function.

Immediately post-photostress, a continuous and timed cycle of foveal sensitivity measurements were conducted and recorded for each subject. The reduction in foveal sensitivity from baseline, along with the time taken to recover to baseline foveal sensitivity, was recorded.

\subsection{Macular pigment optical density}

We used the Macular Densitometer ${ }^{\mathrm{TM}}$, a device developed and originally described by Wooten, Hammond, Land, and Snodderly (1999), to measure MPOD, including its spatial profile across the retina (i.e. $0.25^{\circ}, 0.5^{\circ}, 1.0^{\circ}, 1.75^{\circ}$ and $3^{\circ}$ of retinal eccentricity). The Macular Densitometer ${ }^{\mathrm{TM}}$ uses heterochromatic flicker photometry (HFP) to obtain a valid measure of MPOD at a given retinal location.(Hammond, Wooten, \& Smollon, 2005) This method has recently been refined and is now referred to as customized HFP or cHFP. For a detailed description of this protocol please see recent publications by our research group and others (Loane, Stack, Beatty, \& Nolan, 2007; Nolan et al., 2009; Stringham et al., 2008). One subject (cwit2553) was excluded from analysis due to inability to use the Densitometer to obtain reliable MPOD data.

\subsection{Fundus photography}

Fundus photographs were obtained in both eyes using a NIDEK non-mydriatic fundus camera (AFC-230). Fundus photographs were assessed by an expert eyecare professional to exclude fundoscopically evident retinal pathology.

\subsection{Statistical analysis}

The statistical software package SPSS (version 17) and the statistical programming language $\mathrm{R}$ were used for analysis. It was determined at the outset of the study that a minimum sample size of 91 subjects was required in order to detect an effect size (correlation between two continuous variables) of 0.4 at the $5 \%$ level of significance with high power. However, 121 subjects were recruited into the study in order to allow for dropouts and for other possible analyses, in particular repeated measures analysis.

All continuous variables at baseline exhibited a typical normal distribution. Mean \pm SDs are presented in the text and tables. Comparisons of $\mathrm{A}$ and $\mathrm{P}$ groups at baseline were conducted using independent samples $t$-tests and chi-square analysis, as appropriate.

We conducted repeated measures analysis of MPOD at each retinal eccentricity measured, for each of four study visits using a general linear model approach, with treatment (i.e. A and P) and smoking habits (non-smoker, past and current cigarette smoker) as between-subjects factors. Where appropriate we used the Greenhouse-Geisser correction for violation of sphericity. We used the $5 \%$ level of significance throughout our analysis, without adjustment for multiple testing.

Four visual performance (VP) variables (assessed subjectively by questionnaire) in this study were recorded as percentage change of V4 score compared to V1 score. Repeated measures analysis would not have been appropriate for these, and instead they were analysed using a general linear model with V4 percentage change as the dependent variable and fixed between-subjects factors treatment and smoking habits as explanatory variables.

\section{Results}

\subsection{Baseline findings}

The demographic, lifestyle, dietary and serum carotenoid concentrations, MPOD, and vision data of all 121 subjects recruited into the study, and divided by study arm (i.e. A or P group), are summarized in Table 1 . As seen from this table, there was no

Table 1

Demographic, lifestyle, vision, and macular pigment data at baseline.

\begin{tabular}{|c|c|c|c|c|}
\hline Characteristic & $\begin{array}{l}\text { All } \\
{ }^{\mathrm{a}} n=121\end{array}$ & $\begin{array}{l}\text { A } \\
n=61\end{array}$ & $\begin{array}{l}\mathrm{P} \\
n=60\end{array}$ & ${ }^{* * *}$ Sig. \\
\hline Age & $29 \pm 7$ & $29 \pm 7$ & $29 \pm 6$ & 0.864 \\
\hline Body mass index & $26 \pm 4$ & $26 \pm 4$ & $25 \pm 3$ & 0.736 \\
\hline Best corrected visual acuity & $113 \pm 3$ & $113 \pm 3$ & $112 \pm 3$ & 0.747 \\
\hline \multicolumn{5}{|l|}{ Macular pigment optical density } \\
\hline $0.25^{\circ}$ & $0.5 \pm 0.19$ & $0.49 \pm 0.19$ & $0.51 \pm 0.20$ & 0.458 \\
\hline $0.5^{\circ}$ & $0.4 \pm 0.17$ & $0.39 \pm 0.16$ & $0.41 \pm 0.18$ & 0.425 \\
\hline $1^{\circ}$ & $0.22 \pm 0.13$ & $0.20 \pm 0.12$ & $0.22 \pm 0.15$ & 0.433 \\
\hline $1.75^{\circ}$ & $0.10 \pm 0.11$ & $0.09 \pm 0.10$ & $0.10 \pm 0.11$ & 0.376 \\
\hline $3^{\circ}$ & $0.10 \pm 0.10$ & $0.08 \pm 0.08$ & $0.12 \pm 0.12$ & 0.058 \\
\hline \multicolumn{5}{|l|}{ Dietary carotenoids (mg/day) } \\
\hline Lutein & $1.26 \pm 0.95$ & $1.16 \pm 0.96$ & $1.36 \pm 0.94$ & 0.253 \\
\hline Zeaxanthin & $0.21 \pm 0.12$ & $0.19 \pm 0.10$ & $0.23 \pm 0.14$ & 0.074 \\
\hline \multicolumn{5}{|l|}{ Serum carotenoids $(\mu \mathrm{mol} / \mathrm{L})$} \\
\hline Lutein & $0.60 \pm 0.32$ & $0.57 \pm 0.27$ & $0.62 \pm 0.36$ & 0.399 \\
\hline Zeaxanthin & $0.36 \pm 0.17$ & $0.36 \pm 0.15$ & $0.37 \pm 0.18$ & 0.623 \\
\hline \multicolumn{5}{|l|}{ Sex } \\
\hline Male & 69 & 34 & 35 & \\
\hline Female & 52 & 27 & 25 & 0.773 \\
\hline \multicolumn{5}{|l|}{ Smoking habits ${ }^{\mathrm{b}}$} \\
\hline Never smoked & 73 & 42 & 31 & \\
\hline Ex-smoker & 21 & 11 & 10 & \\
\hline Current smoker & 27 & 8 & 19 & 0.046 \\
\hline \multicolumn{5}{|l|}{ a $n=$ sample size. } \\
\hline
\end{tabular}


significant difference between the $\mathrm{A}$ and $\mathrm{P}$ groups with respect to lifestyle, vision, and MP data, with the exception of a statistically significant difference between these groups for smoking habits $(p=0.046)$. Smoking status was therefore considered as a potential confounding variable and was controlled for throughout repeated measures analysis. The COMPASS baseline findings have already been published in a separate manuscript in this journal and, therefore, are not discussed in the current manuscript (Loughman, Akkali et al., 2010).

\subsection{Longitudinal findings}

\subsubsection{Supplement compliance}

Seventy-six subjects returned tablets, and (based on the number of tablets returned) $94.7 \%$ of these subjects averaged at least one tablet per day. The average number of tablets per day was 1.57 in the A group and 1.65 in the $P$ group, a difference that is not statistically significant (ANOVA, $p=0.32$ ). In comparing change in MPOD and VP variables between A and P groups, therefore, it was not deemed necessary to control for differences in compliance in the two groups.

\subsubsection{Macular pigment optical density}

We conducted repeated measures ANOVA of MPOD, for all retinal eccentricities measured (i.e. at $0.25^{\circ}, 0.5^{\circ}, 1.0^{\circ}, 1.75^{\circ}$, and $3^{\circ}$ ), over time (i.e. over the study period [at V1, V2, V3, and V4, respectively]), using a general linear model approach, with two betweensubjects factors: treatment (A, P) and smoking habits (never, past, current smoker). As seen in Fig. 1, there was a trend (in the A group) towards an increase in MPOD at all eccentricities measured, but this increase was only statistically significant (at the $5 \%$ level) at the more central measured eccentricities (i.e. at $0.25^{\circ}, 0.5^{\circ}$ and $\left.1.75^{\circ}\right)$.

Fig. 2 (obtained from R statistical program) shows MPOD variation at $0.25^{\circ}$ for 20 consecutive individual subjects from each of the $\mathrm{A}$ and $\mathrm{P}$ groups. The graphs are arranged so that those with lowest
MP are in the bottom row, and only subjects who presented for all four visits are displayed.

\subsubsection{Serum concentrations of lutein and zeaxanthin}

We conducted repeated measures analysis of serum concentrations of $\mathrm{L}$ and $\mathrm{Z}$ over time (i.e. over the study period) including all study visits (V1, V2, V3 and V4), using a general linear model approach, with treatment and cigarette smoking as between-subjects factors. As seen in Fig. 3, there was a statistically significant time/ treatment interaction effect for serum concentrations of $\mathrm{L}$, which remained significant ( $p<0.001$, for all) using any of the standard corrections for violation of sphericity. It is clear from the mean plots of Fig. 3, how these significant time/treatment interaction effects came about: serum concentrations of $L$ increased with time in the A group, but remained virtually static in the P group. This time/ treatment effect was significant from V2 (as expected and confirmed using paired t-test analysis between V1 and V2, $p<0.001$ ). There was no statistically significant time or time/treatment interaction effect for serum concentrations of $Z$ over the study period ( $p>0.05$, for all tests); however, there was a trend towards an increase in the A group.

\subsubsection{Visual performance}

While the repeated measures ANOVA presented above is based on findings at all four study visits, it is apparent from the graphs (Figs. 1 and 2) that the largest differences in MPOD between A and P subjects are between V1 and V4. The analysis of VP variables which follows is, therefore, confined to V1 and V4 only (controlling for between-subjects factors: treatment and smoking habits).

Using repeated measures ANOVA or a general linear model, as appropriate, we report a statistically significant time/treatment effect in only one measure of VP, namely "daily tasks comparative analysis" assessed subjectively $(p=0.03)$; whereas all other measures of VP were statistically non-significant $(p>0.05$, for all) [see Table 2].
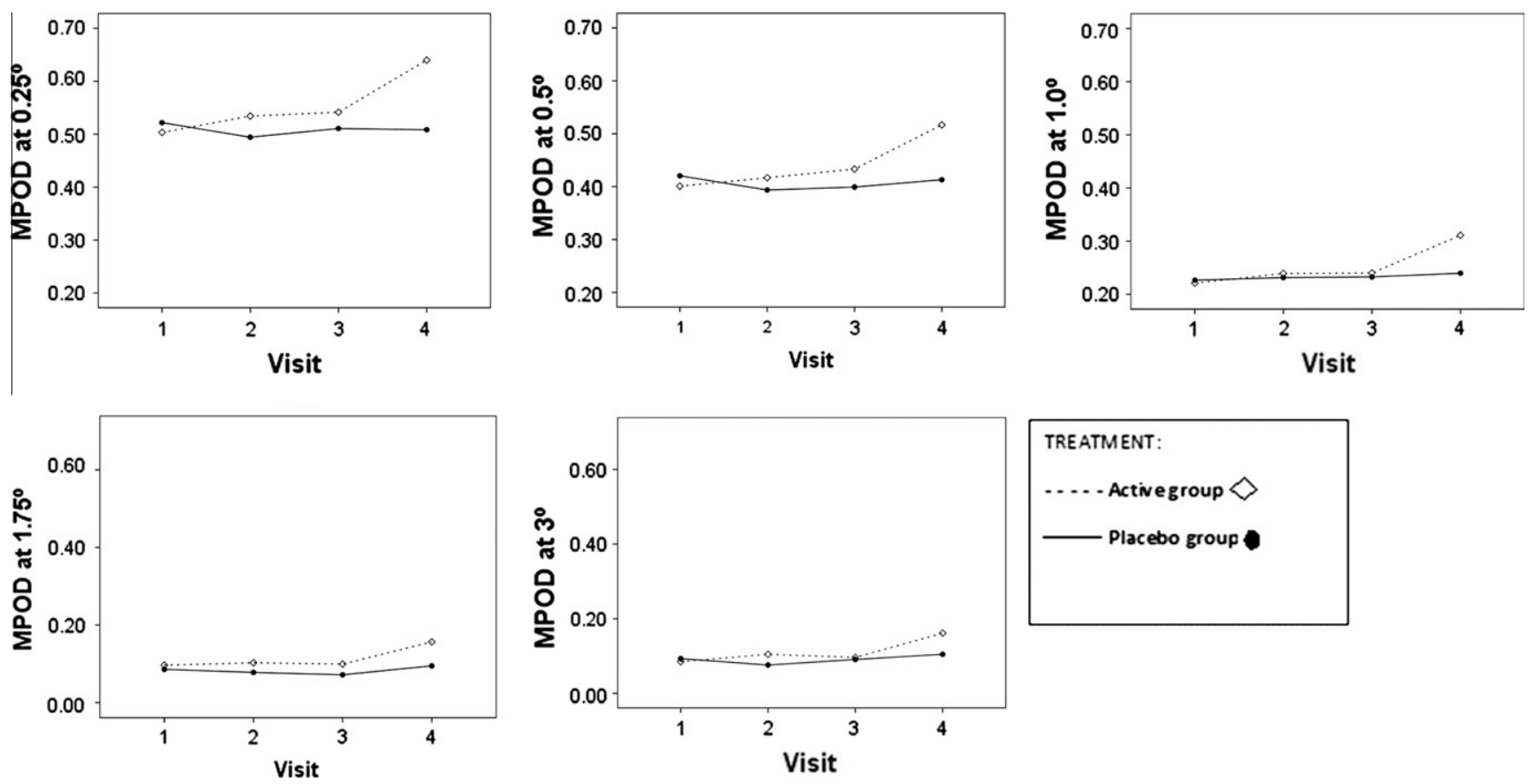

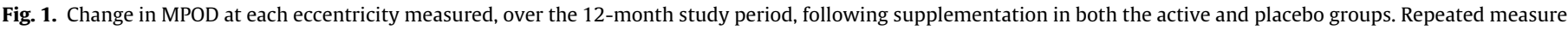

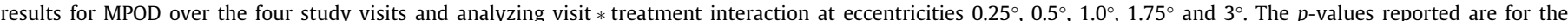

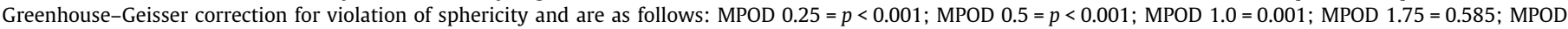
$3.0=0.103$. Subjects were assessed at baseline, 3,6 , and 12 months (V1, V2, V3 and V4, respectively). 

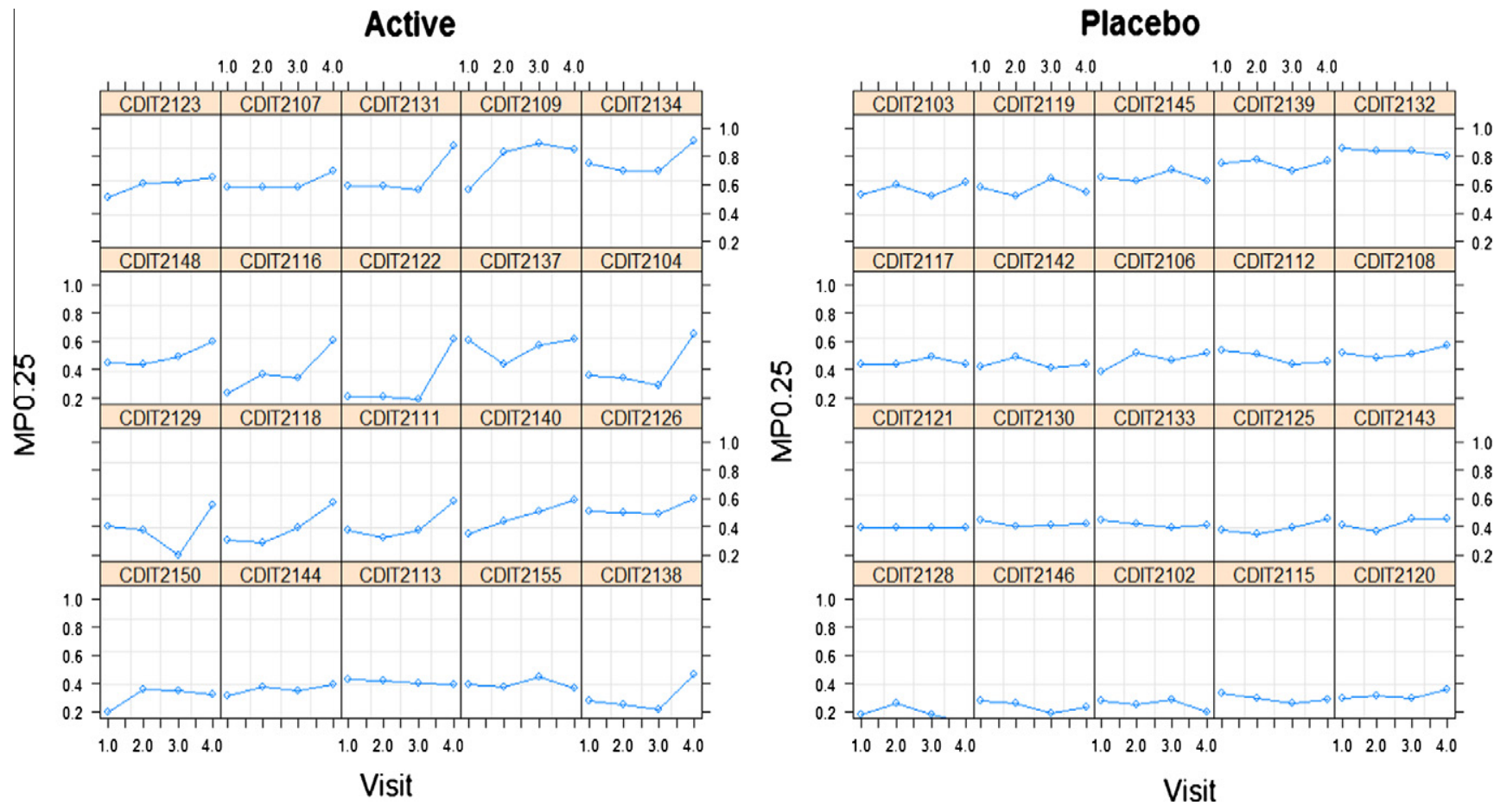

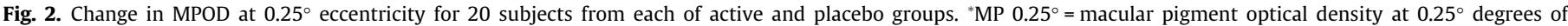
eccentricity

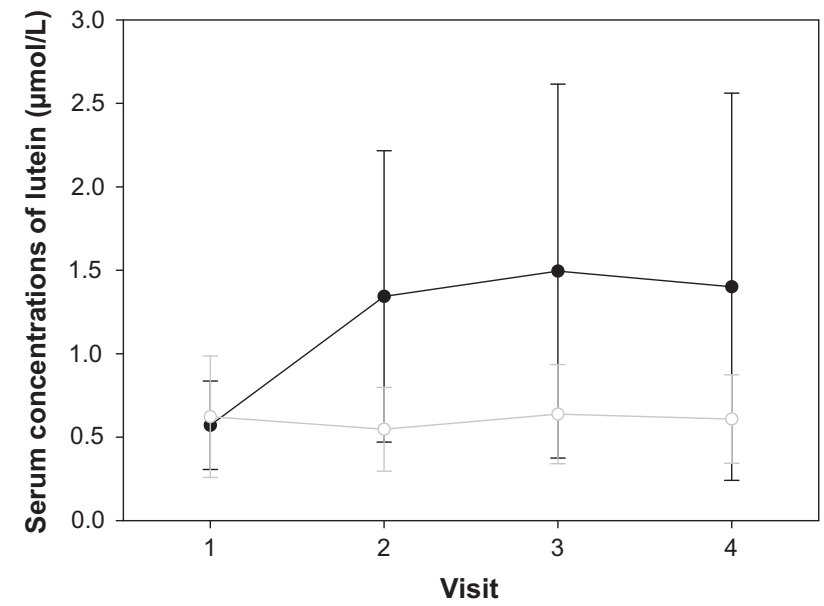

Fig. 3. Change in serum concentrations of lutein over the 12-month study period, following supplementation in both the active and placebo groups. Mean $( \pm S D)$ serum concentrations of lutein were quantified by high-performance liquid chromatography at baseline, 3, 6, and 12 months (V1, V2, V3 and V4, respectively)

\subsubsection{Visual performance differences: low MPOD versus high MPOD subjects}

We investigated whether subjects with high MPOD had significantly better VP scores than subjects with low MPOD following supplementation. We based this investigation, for the most part, on MPOD at $0.25^{\circ}$ at V4. We used tertiles for V4 MPOD at $0.25^{\circ}$ eccentricity to create low, medium and high MPOD groups, and then compared the low and high groups on a variety of VP measures assessed. The low group consisted of 31 subjects with V4 MPOD at or below 0.46 optical density and the high MPOD group had 29 subjects with V4 MPOD at or above 0.69 optical density (Fig. 4). Table 3 presents results for VP measures which differ significantly between these low and high MPOD groups. Table 3 also presents the corresponding results for V1. It should be noted that differences in these VP measures at V1 were not, in general, statistically significant.

\section{Discussion}

COMPASS is a randomized placebo-controlled clinical trial of oral supplementation with a formulation containing the macular carotenoids ( $\mathrm{L}$ and $\mathrm{Z}$ ) and co-antioxidants versus placebo in young normal subjects The pre-specified hypothesis was that supplementation, and consequential MPOD augmentation, would result in improved visual performance and/or comfort in those randomized to the A arm when compared with the P arm, by 12 months.

COMPASS was designed to investigate whether augmentation of MP results in enhancement of visual performance and/or experience, regardless of the mechanism(s) whereby any such improvements may be realized. The optical and neuroprotective hypotheses around MP, which have been discussed previously by Reading and Weale (1974), later by Nussbaum et al. (1981) and are extended here, have generated interest amongst macular pigment scientists, evident in a recent review (Loughman, Davison, Nolan, Akkali, \& Beatty, 2010). In brief, some authors have suggested that MP may be important for visual performance and/or experience by at least one of a number of mechanisms, including the reduction of the effects of chromatic aberration, light scatter, higher order aberrations, and plane polarization of light (Loughman, Davison et al., 2010; Walls \& Judd, 1933). Importantly, however, and in theory at least, the macular carotenoids have the capacity to confer these optical advantages because of their light-filtering and dichroic properties and because of their central location within the retina and crystalline lens.

An additional consideration in relation to any trial investigating the impact of MP augmentation on visual performance and experience is the potential beneficial effect of MP on neurophysiological health. For example, the majority of studies investigating the effects of MP augmentation in ocular disease, including AMD 
Table 2

Repeated measures assessment of all VP measures in COMPASS.

\begin{tabular}{|c|c|c|}
\hline Visual performance measure & Sub-measure/device & $\begin{array}{l}p- \\
\text { value }\end{array}$ \\
\hline Glare disability & $\begin{array}{l}\text { Medium glare (Optec) } \\
1.5 \mathrm{cpd} \\
3.0 \mathrm{cpd} \\
6.0 \mathrm{cpd} \\
12.0 \mathrm{cpd} \\
18.0 \mathrm{cpd}\end{array}$ & $\begin{array}{l}0.58 \\
0.94 \\
0.65 \\
0.96 \\
0.49\end{array}$ \\
\hline Glare disability & $\begin{array}{l}\text { High glare (Optec) } \\
1.5 \mathrm{cpd} \\
3.0 \mathrm{cpd} \\
6.0 \mathrm{cpd} \\
12.0 \mathrm{cpd} \\
18.0 \mathrm{cpd}\end{array}$ & $\begin{array}{l}0.19 \\
0.99 \\
0.89 \\
0.41 \\
0.86\end{array}$ \\
\hline Glare questionnaire & $\begin{array}{l}\text { Glare comparative analysis } \\
\text { Glare change analysis } \\
\text { Glare situational analysis } \\
\text { Glare subject satisfaction score }\end{array}$ & $\begin{array}{l}0.32 \\
0.88 \\
0.74 \\
0.51\end{array}$ \\
\hline Visual acuity & BCVA (Thomson Chart) & 0.16 \\
\hline Visual acuity questionnaire & $\begin{array}{l}\text { Acuity comparative analysis } \\
\text { Acuity change analysis } \\
\text { Acuity situational analysis } \\
\text { Acuity subject satisfaction score }\end{array}$ & $\begin{array}{l}0.08 \\
0.15 \\
0.14 \\
0.59\end{array}$ \\
\hline Daily tasks questionnaire & $\begin{array}{l}\text { Daily tasks comparative analysis } \\
\text { Daily tasks change analysis } \\
\text { Daily tasks situational analysis } \\
\text { Daily tasks subject satisfaction } \\
\text { score }\end{array}$ & $\begin{array}{l}0.03^{*} \\
0.21 \\
0.27 \\
0.41\end{array}$ \\
\hline $\begin{array}{l}\text { Light-dark adaptation } \\
\text { questionnaire }\end{array}$ & $\begin{array}{l}\text { Light-dark comparative analysis } \\
\text { Light-dark change analysis } \\
\text { Light-dark situational analysis } \\
\text { Light-dark subject satisfaction } \\
\text { score }\end{array}$ & $\begin{array}{l}0.35 \\
0.15 \\
0.75 \\
0.56\end{array}$ \\
\hline Mesopic contrast sensitivity & $\begin{array}{l}\text { F.A.C.T. (Optec) } \\
1.5 \mathrm{cpd} \\
3.0 \mathrm{cpd} \\
6.0 \mathrm{cpd} \\
12.0 \mathrm{cpd} \\
18.0 \mathrm{cpd}\end{array}$ & $\begin{array}{l}0.72 \\
0.77 \\
0.84 \\
0.66 \\
0.5\end{array}$ \\
\hline Mesopic contrast sensitivity & $\begin{array}{l}\text { Metropsis } \\
1.0 \mathrm{cpd} \\
4.1 \mathrm{cpd} \\
7.5 \mathrm{cpd} \\
11.8 \mathrm{cpd} \\
20.7 \mathrm{cpd}\end{array}$ & $\begin{array}{l}0.54 \\
0.79 \\
0.82 \\
0.18 \\
0.08\end{array}$ \\
\hline Photopic contrast sensitivity & $\begin{array}{l}\text { Metropsis } \\
1.0 \mathrm{cpd} \\
4.1 \mathrm{cpd} \\
7.5 \mathrm{cpd} \\
11.8 \mathrm{cpd} \\
20.7 \mathrm{cpd}\end{array}$ & $\begin{array}{l}0.95 \\
0.42 \\
0.31 \\
0.19 \\
0.87\end{array}$ \\
\hline Critical flicker fusion frequency & Densitometer & 0.3 \\
\hline Foveal sensitivity & Humphrey perimeter & 0.93 \\
\hline
\end{tabular}

$\mathrm{VP}=$ visual performance; ${ }^{* *}$ sig. = probability significance value.

Four VP variables in this study were recorded as percentage change of V4 score compared to V1 score. Repeated measures analysis would not have been appropriate for these, and instead they were analysed using a general linear model with V4 percentage change as the dependent variable and fixed between-subjects factors treatment and smoking as explanatory variables.

(summarized by Loughman, Davison et al. (2010)), have reported a beneficial effect on vision, and such findings are probably attributable to the neuroprotective, as opposed to the optical, properties of these intracellular compounds. These studies have traditionally employed basic psychophysical outcome measures, including visual acuity and contrast sensitivity, and as such have not included stimuli likely to reveal improvements facilitated solely by image enhancement attributable to the optical properties of this pigment.
The study formulation used in COMPASS, in addition to L and Z, contained the co-antioxidants vitamin C, vitamin E, zinc and selenium. In contrast to the capacity to measure subjects' retinal response to supplementation with the macular carotenoids (i.e. by measuring MP) it was not possible to assess, or quantify, subjects' response to supplementation with the above named co-antioxidants. It is important to note that, as seen in the age-related eye disease study (AREDS) (Kassoff \& The AREDS research group, 2001), that these antioxidants may have contributed to any benefits reported in visual performance in the current study.

Interestingly, several studies have reported, amongst normal subjects, findings which suggest that MP may play a key role in visual health through a complex interplay between the optical, neurological and physiological mechanisms underlying vision. These observations include (a) better critical flicker fusion frequency (CFF) in the presence of higher MPOD (Hammond \& Wooten, 2005), (b) associations between high MPOD and crystalline lens transparency and cataract formation (Brown et al., 1989; ChasanTaber et al., 1999; Hammond, Wooten, \& Snodderly, 1997), (c) the presence of $\mathrm{L}$ and $\mathrm{Z}$ in substantial concentrations in the primary visual cortex (Craft, Haitema, Garnett, Fitch, \& Dorey, 2004) and (d) higher pattern electroretinogram (PERG) P50 amplitudes and better dark adapted cone sensitivities in association with higher MPOD (Carboni, Forma, Mutolo, Jennings, \& Iannaccone, 2010) (Carboni et al., 2010 ARVO Abstract 1293-A105).

The randomized design of COMPASS resulted in desirable baseline similarity between $\mathrm{A}$ and $\mathrm{P}$ groups on possible confounding variables, with the exception of smoking habits (which was controlled for throughout analysis, as appropriate). Significant efforts were made to encourage compliance during the study, and based on the number of tablets returned, we calculated that $95 \%$ of subjects averaged at least one tablet per day, with the average number of tablets consumed per day statistically comparable between the $\mathrm{A}$ and $\mathrm{P}$ groups (at around 1.6 tablets per day).

Consistent with the positive tablet compliance, on average, serum $L$ concentrations increased significantly over the course of the study in the A group with no significant change observed in the $P$ group. Indeed, despite the slight drop in mean serum L concentrations between V3 and V4 in the A group, L concentrations more than doubled in the A group over the course of the study. This finding is consistent with other and recent $\mathrm{L}$ interventional studies (Bone \& Landrum, 2010; Trieschmann et al., 2007). However, while average serum $L$ concentrations significantly increased in the A group and remained stable in the $\mathrm{P}$ group, it is important to point out that 9 (23\%) of the A group showed negative or zero change in serum L concentrations. This "non-response" to L supplementation in serum is consistent with an observation by Hammond et al. in 1997 who reported that one subject (out of 11 measured) demonstrated no significant change in serum concentrations of L following consumption of $\sim 12 \mathrm{mg}$ of $\mathrm{L}$ per day over a 15 week study period (albeit L consumption in that study was achieved from diet [e.g. spinach and corn] and not from dietary supplements [as in the current study]). To explain the high percentage of serum nonresponse in the current study, we propose the following possibilities: non-compliance with respect to consumption of the study tablet in these subjects: possible attenuation of the gastrointestinal absorption of supplemental $\mathrm{L}$ and $\mathrm{Z}$ if the subject fails to take the study tablet in the presence of synchronously ingested fat or oil (importantly, subjects were instructed to consume the daily dose of two tablets with a meal to facilitate the bioavailability of L from the tablet). Indeed, it has been shown that the amount of fat in a person's diet significantly affects the absorption of L ester and its bioavailability, and given that the tablet used in the current study was a film coated tablet not containing oil, failure to consume the study formulation in the presence of fat and/or oil (i.e. with a meal) could significantly impact on the bioavailability of $\mathrm{L}$ (Roodenburg, 


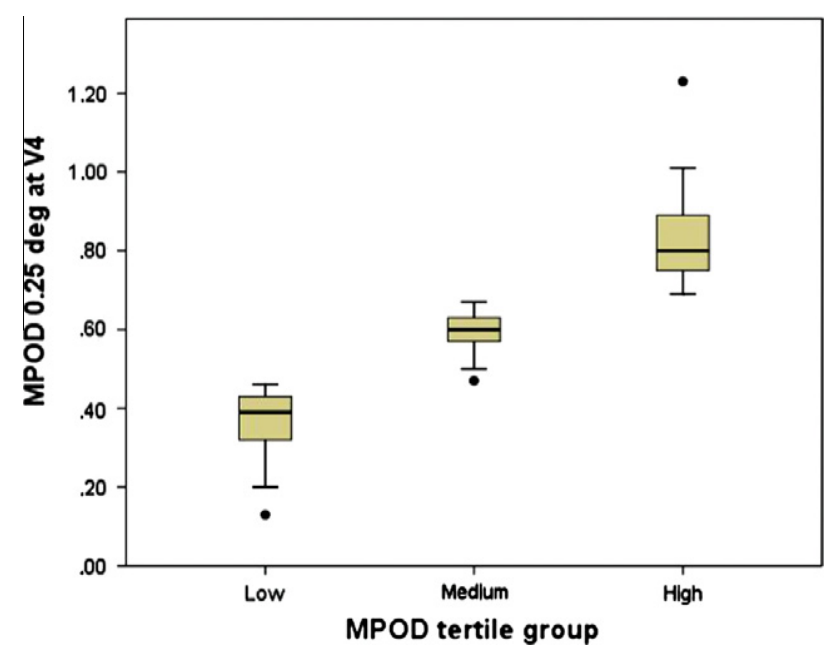

Fig. 4. Boxplots of V4 MPOD at $0.25^{\circ}$ showing range of values for each tertile group. ${ }^{*} \mathrm{MPOD} 0.25^{\circ}$ at visit $4=$ macular pigment optical density at $0.25^{\circ}$ degrees of eccentricity at visit four (12-months) presented for each tertile boxplot. Low, medium and high boxplots represent low tertile group, medium tertile group and high tertile groups with respect to MPOD measured at $0.25^{\circ}$ degrees of eccentricity. Black dots represent extreme values (outliers).

Leenen, van het Hof, Weststrate, \& Tijburg, 2000). Mean serum concentrations of $\mathrm{Z}$ also increased in the $\mathrm{A}$ group, but the increase was not statistically significant, probably due to the low concentration of this carotenoid in the study formulation ( $\sim 1 \mathrm{mg} /$ day).

Central MPOD increased significantly in the A group over the 12-month study period and remained stable in the P group. However, the observed increase in central MPOD in the A group only became apparent (significantly) at 12 months (whereas, as seen above, serum concentrations of $\mathrm{L}$ were significantly augmented in the A group at three months). This finding is consistent with previously published studies reporting slow uptake of $\mathrm{L}$ by the retina (Bone, Landrum, Guerra, \& Ruiz, 2003; Johnson et al., 2000), and inconsistent with others (Connolly et al. (2010)). However, it should be noted that the retinal uptake in our study was much slower than any of these previously published studies. For example, Bone et al. report that no significant change in MP was seen until after day 40 following supplementation with $\mathrm{L}$ and $\mathrm{Z}$ with up to $30 \mathrm{mg} /$ day of each carotenoid and Johnson et al. report a significant increase in MP after 4 weeks of consuming $60 \mathrm{~g} /$ day spinach and $150 \mathrm{~g} /$ day corn. However, the reason(s) for the difference seen between studies may be due to any (or a combination of) the following factors: dose of $\mathrm{L}$ and $\mathrm{Z}$ consumed per day; type of $\mathrm{L}$ and $\mathrm{Z}$ in the supplement (e.g. free versus ester) matrix in which carotenoids are consumed (e.g. oil versus micro-encapsulated); whether consumed alone or in the presence of other antioxidants; poor serum response to the supplement; non-compliance to the study supplement. Further, and detailed, study on this interesting topic is merited.

The average increase seen in the A group at $0.5^{\circ}$ of retinal eccentricity (the standard and most commonly measured and reported MPOD eccentricity) over the 12-month study period was $0.11 \pm 0.005$ optical density, which is comparable to the findings of Trieschmann et al. who reported an average increase in MP of $0.10 \pm 0.009$ optical density where they measured MPOD by 2wavelength autofluorescence. Interestingly, Trieschmann et al. used the same study formulation (daily consumption of $12 \mathrm{mg}$ of $\mathrm{L}$ provided as ester) over a 12-month study period as that used in the current study, but by delivering four tablets per day (each containing $3 \mathrm{mg}$ of L ester), whereas the current study achieved a daily consumption of $12 \mathrm{mg}$ of $\mathrm{L}$ ester by delivering two tablets per day (Trieschmann et al., 2007). Unlike the findings reported by Trieschmann et al., we report that the biggest gain in MPOD in the A group did not, in general, occur in subjects with lowest baseline MPOD values. However, consistent with the data reported by Trieschmann et al., who reported that 20 (21\%) of 92 subjects assessed were retinal non-responders (at $0.5^{\circ}$ ), we found that eight (17\%) of the A group at $0.25^{\circ}$ and nine $(20 \%)$ of the A group at $0.5^{\circ}$ showed negative or zero change in MP at 12 months.

In contrast with the MP measures discussed above, the VP measures assessed in the current study did not, in general, improve significantly over time in the A group. This would, superficially at least, seem to be at odds with the optical and visual health hypotheses of MP's function. Indeed, it is important to emphasise that, of all the VP measures assessed, and reported on, in COMPASS (48 variables in total; see Table 2 ) we report a statistically significant result for only one measure, namely "daily tasks comparative analysis", assessed subjectively. It is possible, therefore, as data from the current study suggest, that supplementation with the macular carotenoids, and consequential MP augmentation, has no major impact on visual performance and/or experience in young normal subjects (our primary research question and the main study hypothesis). This is, however, at odds with previous reports with respect to the impact of MPOD augmentation on glare disability (Stringham \& Hammond, 2005, 2008). This discrepancy with earlier findings may be explained, at least partly, by two fundamental differences between the relevant studies. Firstly, COMPASS was designed to evaluate glare disability under conditions approximating normal environmental experience. As such, testing was conducted using natural pupils, which typically constrict under glare conditions, and therefore confer protection against the effects of glare. The Maxwellian view system employed in other studies does not

Table 3

Comparing visual performance measures between low and high macular pigment optical density groups at visit 4 and visit 1 .

\begin{tabular}{|c|c|c|c|c|c|c|}
\hline \multirow[t]{2}{*}{ Visual performance variable } & \multicolumn{3}{|l|}{ Visit 4} & \multicolumn{3}{|l|}{ Visit 1} \\
\hline & MP group ${ }^{a}$ & Mean $( \pm S D)$ & Sig. & MP group & Mean $( \pm S D)$ & Sig. \\
\hline \multirow{2}{*}{ Best corrected visual acuity } & High & $113(3)$ & & High & $113(3)$ & \\
\hline & Low & $111(4)$ & 0.038 & Low & $112(3)$ & 0.045 \\
\hline \multirow[t]{2}{*}{ Mesopic CS at $1.5 \mathrm{cpd}$ under high glare ${ }^{\mathrm{b}}$} & High & $28.6(15.8)$ & & High & $22.1(11.6)$ & \\
\hline & Low & $21.2(11.2)$ & 0.042 & Low & $19.8(8.2)$ & 0.337 \\
\hline \multirow[t]{2}{*}{ Light/dark adaptation comparative analysis ${ }^{c}$} & High & $70.3(17.4)$ & & High & $62.2(13.2)$ & \\
\hline & Low & $60.6(13.1)$ & 0.018 & Low & $60.6(14.7)$ & 0.624 \\
\hline \multirow[t]{2}{*}{ Mesopic contrast sensitivity at $20.7 \mathrm{cpd} \Omega$} & High & $54.7(17.4)$ & & High & $57.1(15.0)$ & \\
\hline & Low & $62.9(10.9)$ & 0.035 & Low & $59.2(12.1)$ & 0.523 \\
\hline
\end{tabular}

a $\mathrm{MP}$ group = macular pigment optical density group tertile for $0.25^{\circ}$ eccentricity: high = top tertile, low = bottom tertile .

b Mesopic CS at $1.5 \mathrm{cpd}$ under high glare = night-time contrast sensitivity at low spatial frequencies assessed under high glare conditions.

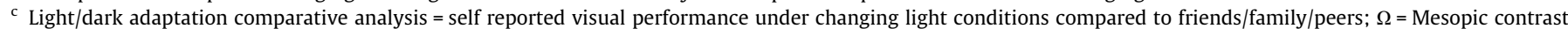
sensitivity at $20.7 \mathrm{cpd}=$ night time contrast sensitivity measured at high spatial frequencies. 
allow normal pupillary response, so, while MP was shown to impact glare disability under these conditions, it is not clear whether the effect would have remained if a pupillary response had been allowed, which would have caused a variable reduction in retinal illuminance proportional to the magnitude of the pupillary response. Secondly, our findings can only be applied to the stimulus and glare intensity settings employed here, which, although informed by a detailed pilot study, are less comprehensive than the variable glare annulus intensity employed by Stringham \& Hammond.

Kvansakul et al. (Kvansakul et al., 2006) conducted a study to evaluate the effect of MP supplementation on mesopic contrast acuity thresholds (CAT) in normal subjects.(Kvansakul et al., 2006) They reported a significant and beneficial effect of MP supplementation on mesopic CAT that was not evident in their placebo group, their findings therefore appearing to be at odds with those of the current study, probably reflecting a number of differences between the two studies in terms of methodology and design [e.g. stimuli, illumination levels $\left(1 \mathrm{cdm}^{-2}\right.$ vs $\left.3 \mathrm{cdm}^{-2}\right)$, etc.]. Also, the design by Kvansakul et al. did not incorporate longitudinal evaluation of MPOD, which was measured only at the final visit (interestingly the CATs reported by Kvansakul showed no correlation with MPOD). Furthermore, contrast acuity thresholds were not measured at baseline, but only after six months of supplementation and then again at the final 12 month visit. One cannot, therefore, draw meaningful conclusions with respect to the relationship, if any, between their mesopic CAT findings and MPOD, as there is no record of change in MPOD over their study period. A final point relates to the sample sizes of the two studies, the investigation by Kvansakul et al. being based on a placebo group of only five subjects and three groups of subjects receiving supplementation (containing three, five and five subjects respectively) and is thus not comparable with the COMPASS trial, involving 121 subjects.

There are however, a number of plausible explanations for the absence of any significant influence of MP augmentation on visual performance in our study. Firstly, it should be noted that the majority of study participants exhibited average to high central MPOD pre-supplementation. Indeed, only a small number of subjects ( $24 \%$ ) were found to have central MPOD (at $0.5^{\circ}$ eccentricity) less than 0.30 at baseline. Importantly, it has been suggested previously that MPOD levels greater than 0.30 might be superfluous to visual performance requirements (Reading \& Weale, 1974), due to the non-linear nature of the effect of MP on vision. Furthermore, the increase in MPOD observed in the A group did not become apparent until the final 12 month visit, and was relatively modest with an average increase of $0.11 \pm 0.005$ optical density (at $0.5^{\circ}$ eccentricity), and unlike the findings reported by Trieschmann et al., subjects (in the A group) in the current study with the lowest MP at baseline did not, in general, demonstrate the biggest increase in MPOD levels following supplementation with the study formulation. Indeed, even after 12-months of supplementation with $12 \mathrm{mg}$ of L per day, over $15 \%$ of subjects in the A group retained central MPOD (at $0.5^{\circ}$ eccentricity) values below 0.3 optical density. In other words, it is possible that the MP augmentation achieved in the current study was not sufficient (in an adequate number of subjects) to impact on visual performance, and that a greater increase in MPOD, particularly in the group with lowest baseline MPOD, might be required to elicit an improvement in visual performance. Also, as mentioned above, it is also likely that a significant number of subjects in the current study already had (at baseline) sufficient MP for optimal, measurable, and appreciable visual performance (i.e. $75 \%$ of subjects in the A group had baseline MP values $\geqslant 0.3$ optical density) and therefore may explain, at least in part, the failure of the current study to demonstrate an improvement in VP following supplemental $\mathrm{L}$.
In addition, the nature of the tests employed for visual performance testing in COMPASS also merits consideration and discussion. The investigators strategically chose to use tests that were either typically available in the average consulting room (to ensure applicability of findings to clinical practice), or designed to replicate typical environmental conditions. As such, most of the tests did not contain substantial amounts of short wavelength light maximally absorbed by MP. The typical office or home environment (where the majority of us spend most of our time), does not have many short wave dominated light sources. Our results might, therefore, suggest that subjects' MP levels pre-supplementation were sufficient for optimal visual performance in this type of environment. Our results, therefore, cannot be extrapolated to short wave dominated visual scenes, such as against the background of a bright blue sky, which is difficult to replicate in an ecologically valid way. Importantly, the changing nature of internal and device lighting systems, such as the increased use of LED systems, and xenon car headlights, are extending our exposure to short wave light sources, and may enhance the applicable relevance of MP for visual performance.

However, given that our study subjects showed an extensive range of MP values, we considered it meaningful to compare VP and comfort measures for subjects with high MP (upper tertile) versus subjects with low MP (lower tertile). We made these comparisons at baseline and also at V4. At V1, the subjects in the low MP group (for central MP at $0.25^{\circ}$ ) were below 0.42 optical density, whereas subjects in the high MP group (for central MP at $0.25^{\circ}$ ) were above 0.59 optical density. At V4, the corresponding figures for low and high groups were 0.46 and 0.67 optical density. Supplementation with $\mathrm{L}$, therefore, appears to have widened the gap in MP between the lower and upper tertiles. Of interest, at V4 we report statistically significant differences in some important VP measures, between lower and upper MP tertile groups, which were not present at $\mathrm{V} 1$.

The most significant finding is that of a $\sim 30 \%$ greater CS under high glare conditions in those with highest MPOD following supplementation. Interestingly, of all the tests employed in COMPASS, the glare source contained the most substantial amount of short wave light (white LEDs used to generate glare contain a single "blue" peak around $460 \mathrm{~nm}$ ). These results therefore would seem to corroborate previous findings which suggest a role for MP in the attenuation of glare disability (Stringham and Hammond, 2007, 2008; Stringham et al., 2004), and furthermore would seem to extend those findings to suggest that MP augmentation is beneficial for visual performance under glare conditions, even under the natural pupil conditions employed here. This finding and hypothesis is also supported by the results of the visual performance questionnaire. Subjects in the A group reported comparatively, and statistically significantly, better visual performance for daily visual tasks (including night driving against oncoming headlights). Furthermore, in the tertile analysis, those with the highest MP reported comparatively, and statistically significantly, better, capacity to deal with sudden changes in illumination (light/dark adaptation).

In conclusion, we report that a significant increase in central MP following L supplementation does not, in general, impact on VP in young normal subjects, and our pre-specified hypothesis that MP augmentation would result in improved VP and/or comfort by 12 months, in those randomized to the A arm, remains unproven. However, subjects with high MP following L supplementation demonstrate visual benefits with respect to glare disability and mesopic CS. Further study into MP and its relationship with VP is warranted to enhance our understanding of this pigment's role. However, in order to investigate the impact of MP augmentation on visual performance, the findings of our study suggest that we should direct our attention to a) subjects with low baseline central 
MP levels, b) subjects with suboptimal visual performance and c) subjects with symptoms of glare disability.

\section{Grant information}

Study supported by Bausch \& Lomb Inc. and Enterprise Ireland, under the Innovation Partnerships Programme.

\section{Acknowledgments}

We would like to acknowledge Enterprise Ireland and the Innovation Partnership of Bausch \& Lomb Inc. who supported this research.

\section{Appendix A. Supplementary material}

Supplementary data associated with this article can be found, in the online version, at doi:10.1016/j.visres.2010.12.016.

\section{References}

Algvere, P. V., Marshall, J., \& Seregard, S. (2006). Age-related maculopathy and the impact of blue light hazard. Acta Ophthalmologica Scandinavica, 84(1), 4-15.

Bartlett, H. E., \& Eperjesi, F. (2008). A randomised controlled trial investigating the effect of lutein and antioxidant dietary supplementation on visual function in healthy eyes. Clinical Nutrition, 27(2), 218-227.

Beatty, S., Koh, H. H., Henson, D., \& Boulton, M. (2000). The role of oxidative stress in the pathogenesis of age-related macular degeneration. Survey of Ophthalmology, 45(2), 115-134

Bone, R. A., \& Landrum, J. T. (2010). Dose-dependent response of serum lutein and macular pigment optical density to supplementation with lutein esters. Archives of Biochemistry and Biophysics.

Bone, R. A., Landrum, J. T., \& Cains, A. (1992). Optical-density spectra of the macular pigment in vivo and in vitro. Vision Research, 32(1), 105-110.

Bone, R. A., Landrum, J. T., Guerra, L. H., \& Ruiz, C. A. (2003). Lutein and zeaxanthin dietary supplements raise macular pigment density and serum concentrations of these carotenoids in humans. Journal of Nutrition, 133(4), 992-998.

Bone, R. A., Landrum, J. T., Hime, G. W., Cains, A., \& Zamor, J. (1993). Stereochemistry of the human macular carotenoids. Investigative Ophthalmology and Visual Science, 34(6), 2033-2040.

Brown, E. D., Micozzi, M. S., Craft, N. E., Bieri, J. G., Beecher, G., Edwards, B. K., et al (1989). Plasma carotenoids in normal men after a single ingestion of vegetables or purified beta-carotene. American Journal of Clinical Nutrition, 49(6), 1258-1265.

Carboni, G., Forma, G., Mutolo, M. G., Jennings, B. J., \& Iannaccone, A. (2010). Crosssectional correlations between macular pigment optical density (MPOD) and measures of macular function. Investigative Ophthalmology and Visual Science 1293-A105. Ref Type: Abstract.

Chasan-Taber, L., Willett, W. C., Seddon, J. M., Stampfer, M. J., Rosner, B., Colditz, G. A., et al. (1999). A prospective study of carotenoid and vitamin A intakes and risk of cataract extraction in US women. American Journal of Clinical Nutrition, $70(4), 509-516$.

Congdon, N., O'Colmain, B., Klaver, C. C., Klein, R., Munoz, B., Friedman, D. S., et al. (2004). Causes and prevalence of visual impairment among adults in the United States. Archives of Ophthalmology, 122(4), 477-485

Connolly, E. E., Beatty, S., Thurnham, D. I., Loughman, J., Howard, A. N., Stack, J., et al. (2010). Augmentation of macular pigment following supplementation with all three macular carotenoids: An exploratory study. Current Eye Research, 35(4), 335-351.

Craft, N. E., Haitema, T. B., Garnett, K. M., Fitch, K. A., \& Dorey, C. K. (2004). Carotenoid, tocopherol, and retinol concentrations in elderly human brain. Journal of Nutrition Health and Aging, 8(3), 156-162.

Dhalla, M. S., \& Fantin, A. (2005). Macular photostress testing: Sensitivity and recovery with an automated perimeter. Retina, 25(2), 189-192.

Dhalla, M. S., Fantin, A., Blinder, K. J., \& Bakal, J. A. (2007). The macular automated photostress test. American Journal of Ophthalmology, 143(4), 596-600.

Engles, M., Wooten, B., \& Hammond, B. (2007). Macular pigment: A test of the acuity hypothesis. Investigative Ophthalmology and Visual Science, 48(6), 2922-2931.

Fletcher, A. E., Bentham, G. C., Agnew, M., Young, I. S., Augood, C., Chakravarthy, U., et al. (2008). Sunlight exposure, antioxidants, and age-related macular degeneration. Archives of Ophthalmology, 126(10), 1396-1403.

Hammond, B. R., Jr., \& Wooten, B. R. (2005). CFF thresholds: Relation to macular pigment optical density. Ophthalmic and Physiological Optics, 25(4), 315-319.

Hammond, B. R., Jr., Wooten, B. R., \& Smollon, B. (2005). Assessment of the validity of in vivo methods of measuring human macular pigment optical density. Optometry and Vision Science, 82(5), 387-404

Hammond, B. R., Wooten, B. R., \& Snodderly, D. M. (1997). Density of the human crystalline lens is related to the macular pigment carotenoids, lutein and zeaxanthin. Optometry and Vision Science, 74(7), 499-504.
Hemenger, R. P. (1982). Dichroism of the macular pigment and Haidinger's brushes. Journal of the Optical Society of America, 72(6), 734-737.

Hirsch, J., \& Curcio, C. A. (1989). The spatial resolution capacity of human foveal retina. Vision Research, 29(9), 1095-1101.

Hitchcock, E. M., Dick, R. B., \& Krieg, E. F. (2004). Visual contrast sensitivity testing: A comparison of two FA.C.T. test types. Neurotoxicology and Teratology, 26(2), 271-277.

Hohberger, B., Laemmer, R., Adler, W., Juenemann, A. G., \& Horn, F. K. (2007) Measuring contrast sensitivity in normal subjects with OPTEC 6500: Influence of age and glare. Graefes Archive for Clinical and Experimental Ophthalmology, 245(12), 1805-1814.

Johnson, E. J., Hammond, B. R., Yeum, K. J., Qin, J., Wang, X. D., Castaneda, C., et al. (2000). Relation among serum and tissue concentrations of lutein and zeaxanthin and macular pigment density. American Journal of Clinical Nutrition, 71(6), 1555-1562.

Kassoff, A.The AREDS research group. (2001). A randomized, placebo-controlled, clinical trial of high-dose supplementation with vitamins $C$ and $E$, beta carotene and zinc for age-related macular degeneration and vision loss: AREDS report no. 8. Archives of Ophthalmology, 119(10), 1417-1436.

Khachik, F., Bernstein, P. S., \& Garland, D. L. (1997). Identification of lutein and zeaxanthin oxidation products in human and monkey retinas. Investigative Ophthalmology and Visual Science, 38(9), 1802-1811.

Kvansakul, J., Rodriguez-Carmona, M., Edgar, D. F., Barker, F. M., Kopcke, W., Schalch, W., et al. (2006). Supplementation with the carotenoids lutein or zeaxanthin improves human visual performance. Ophthalmic and Physiological Optics, 26(4), 362-371.

Loane, E., Nolan, J. M., \& Beatty, S. (2010). The respective relationships between lipoprotein profile, macular pigment optical density, and serum concentrations of lutein and zeaxanthin. Investigative Ophthalmology and Visual Science, 51(11), 5897-5905.

Loane, E., Kelliher, C., Beatty, S., \& Nolan, J. M. (2008). The rationale and evidence base for a protective role of macular pigment in age-related maculopathy. British Journal of Ophthalmology, 92(9), 1163-1168.

Loane, E., Stack, J., Beatty, S., \& Nolan, J. M. (2007). Measurement of macular pigment optical density using two different heterochromatic flicker photometers Current Eye Research, 32(6), 555-564.

Loughman, J., Akkali, M. C., Beatty, S., Scanlon, G., Davison, P. A., O'Dwyer, V., et al. (2010). The relationship between macular pigment and visual performance. Vision Research.

Loughman, J., Davison, P. A., Nolan, J. M., Akkali, M. C., \& Beatty, S. (2010). Macular pigment and its contribution to visual performance and experience. Journal of Optometry, 3(2), 74-90. Ref Type: Journal (Full).

Maoka, T., Arai, A., Shimizu, M., \& Matsuno, T. (1986). The first isolation of enantiomeric and meso-zeaxanthin in nature. Comparative Biochemistry and Physiology B, 83(1), 121-124.

Nebeling, L. C., Forman, M. R., Graubard, B. I., \& Snyder, R. A. (1997a). Changes in carotenoid intake in the United States: The 1987 and 1992 Nationa Health Interview Surveys. Journal of the American Dietetic Association, 97(9), 991-996.

Nebeling, L. C., Forman, M. R., Graubard, B. I., \& Snyder, R. A. (1997b). The impact of lifestyle characteristics on carotenoid intake in the United States: The 1987 National Health Interview Survey. American Journal of Public Health, 87(2), 268-271.

Nolan, J. M., O'Reilly, P., Loughman, J., Stack, J., Loane, E., Connolly, E., et al. (2009). Augmentation of macular pigment following implantation of blue light-filtering intraocular lenses at the time of cataract surgery. Investigative Ophthalmology and Visual Science, 50(10), 4777-4785.

Nussbaum, J. J., Pruett, R. C., \& Delori, F. C. (1981). Historic perspectives. Macular yellow pigment. The first 200 years. Retina, 1(4), 296-310.

O'Connell, E. D., Nolan, J. M., Stack, J., Greenberg, D., Kyle, J., Maddock, L., \& Beatty, S (2008). Diet and risk factors for age-related maculopathy. American Journal of Clinical Nutrition, 87(3), 712-722.

Perry, A., Rasmussen, H., \& Johnson, E. J. (2009). Xanthophyll (lutein, zeaxanthin) content in fruits, vegetables and corn and egg products. Journal of Food Composition and Analysis, 22(1), 9-15

Reading, V. M., \& Weale, R. A. (1974). Macular pigment and chromatic aberration. Journal of the Optical Society of America, 64(2), 231-234.

Rodriguez-Carmona, M., Kvansakul, J., Harlow, J. A., Kopcke, W., Schalch, W. \& Barbur, J. L. (2006). The effects of supplementation with lutein and/or zeaxanthin on human macular pigment density and colour vision. Ophthalmic and Physiological Optics, 26(2), 137-147.

Roodenburg, A. J., Leenen, R., van het Hof, K. H., Weststrate, J. A., \& Tijburg, L. B. (2000). Amount of fat in the diet affects bioavailability of lutein esters but not of alpha-carotene, beta-carotene, and vitamin E in humans. American Journal of Clinical Nutrition, 71(5), 1187-1193.

Roth, H. L., Lora, A. N., \& Heilman, K. M. (2002). Effects of monocular viewing and eye dominance on spatial attention. Brain, 125(Pt 9), 2023-2035.

Sloane, M. E., Ball, K., Owsley, C., Bruni, J. R., \& Roenker, D. L. (1992). The visual activities questionnaire: Developing an instrument for assessing problems in everyday visual tasks. Technical digest, noninvasive assessment of the visual system, topical meeting of the optical society of America.

Sommerburg, O., Keunen, J. E. E., Bird, A. C., \& van Kuijk, F. J. G. M. (1998). Fruits and vegetables that are sources for lutein and zeaxanthin: The macular pigment in human eyes. British Journal of Ophthalmology, 82(8), 907-910.

Stringham, J. M., Fuld, K., \& Wenzel, A. J. (2004). Spatial Properties of Photophobia Investigative Ophthalmology and Visual Science, 45(10), 3838-3848. 
Stringham, J. M., \& Hammond, B. R. Jr., (2005). Dietary lutein and zeaxanthin: Possible effects on visual function. Nutrition Reviews, 63(2), 59-64.

Stringham, J. M., \& Hammond, B. R. Jr., (2007). The glare hypothesis of macular pigment function. Optometry and Vision Science, 84(9), 859-864.

Stringham, J. M., \& Hammond, B. R. (2008). Macular pigment and visual performance under glare conditions. Optometry and Vision Science, 85(2), 82-88.

Stringham, J. M., Hammond, B. R., Nolan, J. M., Wooten, B. R., Mammen, A., Smollon, W., et al. (2008). The utility of using customized heterochromatic flicker photometry (cHFP) to measure macular pigment in patients with age-related macular degeneration. Experimental Eye Research, 87(5), 445-453.

Terzi, E., Buhren, J., Wesemann, W., \& Kohnen, T. (2005). [Frankfurt-Freiburg Contrast and Acuity Test System (FF-CATS). A new test to determine contrast sensitivity under variable ambient and glare luminance levels]. Ophthalmologe, 102(5), 507-513.
Trieschmann, M., Beatty, S., Nolan, J. M., Hense, H. W., Heimes, B., Austermann, U., et al. (2007). Changes in macular pigment optical density and serum concentrations of its constituent carotenoids following supplemental lutein and zeaxanthin: The LUNA study. Experimental Eye Research, 84(4), $718-728$.

Walls, G. L., \& Judd, H. D. (1933). The intra-ocular colour-filters of vertebrates. British Journal of Ophthalmology, 17(12), 705-725.

Winkler, B. S., Boulton, M. E., Gottsch, J. D., \& Sternberg, P. (1999). Oxidative damage and age-related macular degeneration. Molecular Vision, 5(32), 32.

Wooten, B. R., \& Hammond, B. R. (2002). Macular pigment: Influences on visual acuity and visibility. Progress in Retinal and Eye Research, 21(2), 225-240.

Wooten, B. R., Hammond, B. R., Land, R. I. \& Snodderly, D. M. (1999). A practical method for measuring macular pigment optical density. Investigative Ophthalmology and Visual Science, 40(11), 2481-2489. 